\title{
On the Achievable Rate of Three-Node Cognitive Hybrid Wireless Networks
}

\author{
Kyounghwan Lee and Aylin Yener \\ Wireless Communications and Networking Laboratory \\ Department of Electrical Engineering \\ The Pennsylvania State University \\ University Park, PA 16802 \\ kxl251@psu.edu_yener@ee.psu.edu
}

\begin{abstract}
In this paper, we investigate the informationtheoretic performance of the multi-band relay channel which is defined by a source node, a relay node, and a destination node communicating over multiple orthogonal bands. The model is motivated by the vision of hybrid wireless networks where links operating with different communication standards relay information from the source to the destination. We consider hybrid wireless networks, with cognitive and agile radios, where bandwidth and power are the shared resources between different systems. We first provide the capacity bounds and find the optimum resource allocation that maximizes the achievable rate for a simple network. Based on the allocation strategy found, we next study the impact of optimum resource allocation on the construction of a hybrid wireless network, in particular, the scenario where a new source to relay and destination band is added to a classical frequency division relay network. Given the channel conditions of the network, we establish the guidelines on how to allocate resources in order to achieve the higher achievable rates, depending on the relative quality of the available links.
\end{abstract}

\section{INTRODUCTION}

Future wireless networks are expected to enable nodes to communicate over multiple technologies and multiple hops. Recent advances in the development of software defined radios support the vision where cognitive agile radios are employed at each node that utilize multiple standards and communicate seamlessly. Indeed, an intense research effort is being directed towards having multiple communication standards coexist within one system, e.g. the cellular network and IEEE 802.11 WLAN as in [1]. We refer to a group of cognitive nodes capable of employing a number of communication technologies in an effort to find the best multi-hop route between the source-destination pairs, as a hybrid wireless network.

In this paper, we consider a simple hybrid wireless network with a source destination pair, and aim at understanding the bounds of its information theoretic capacity with optimum resource allocation. In particular, we consider a scenario where the source node can communicate over multiple frequency bands to its destination, and a node that overhears the source transmission acts as a relay. We assume that the frequency bands that the source utilizes as well the ones used by the relay node are mutually orthogonal. The different bands are assumed to represent links that operate with different wireless communication standards.
There has been considerable research effort towards characterizing the information theoretic capacity of relay networks in the past [2], [3], which has been rejuvenated with the recent advances in multi-hop ad hoc network architectures [4]-[7]. Cover and El Gamal provide capacity bounds for the Gaussian relay channel, and find the capacity of the degraded Gaussian relay channel [3]. The work of Schein and Gallager investigates capacity upper and lower bounds on the Gaussian parallel relay channel, where two relay channels exist [5]. The study of obtaining an achievable rate region in a relay network of arbitrary size and topology has been investigated in [6]. More recent work considers optimum resource allocation for relay networks to increase the achievable rate and spectral efficiency [8]-[10].

In this paper, we investigate the capacity bounds for a simple, three node hybrid relay network. For the case where the source has two bands, and the relay has a single band available, we find the optimum resource allocation that maximizes the achievable rate. In order to gain insight into the impact of optimum resource allocation on the construction of a hybrid wireless network, we next examine a scenario where a new wireless link is added to the classical frequency division relay network to form a simple hybrid wireless network. We observe that the source node is encouraged to communicate over the best network by dedicating all resources exclusively when the condition of source to relay (SR) link and source to destination (SD) link of the new network is better (or worse) than that of the SD link and the SR link of the current link. Otherwise, it is beneficial to share resource between the current network and the new link to increase the achievable rate.

\section{A. Relation to Previous Work}

Optimum resource allocation for the relay channel has been considered in [8], [9], [11]. The model we consider is inspired by the parallel relay channel [5], [8]. Reference [8] considers the optimum resource allocation problem for the classical frequency division relay channel, where the source transmits in one frequency band, and the relay transmits in one frequency band that is orthogonal to that of the source. We generalize the model in [8] by considering multiple orthogonal channels from the source and the relay node, and investigate the capacity bounds of the resulting multi-band relay channel (MBR) with optimum resource allocation. 
Reference [11] investigates three different half-duplex timedivision based protocols that vary in the degree of broadcasting they employ and the existence of receiver collision. The optimum power and time-slot allocation has been investigated for the protocol with the maximum degree of broadcasting and no receiver collision in [9]. The MBR differs from these models in that multiple orthogonal channels are present from which the receivers experience different noise levels.

\section{The Multi-Band Relay Channel (MBR)}

We consider a three node hybrid wireless network where multiple frequency bands available from the source and the relay are mutually orthogonal. We term this the multi-band relay channel (MBR). In particular, the situation where there are $m$ channels available for the source node and $k-m$ for the relay node, shown in Figure 1 is termed the $(k, m)$-MBR.

We reemphasize that the MBR is the generalization of the relay channel model with two orthogonal bands in [8] to arbitrary number of orthogonal channels from the source and the relay node.

The source node transmits the desired information over $m$ orthogonal channels to the relay node and the destination. The relay node decodes and re-encodes the received data to relay to the destination. The input-output signal model is given by

$$
\begin{aligned}
\tilde{\mathbf{Y}}_{\mathbf{S R}} & =\mathbf{X}_{\mathbf{S}}+\tilde{\mathbf{Z}}_{\mathbf{S R}} \\
\mathbf{Y}_{\mathbf{R D}} & =\tilde{\mathbf{X}}_{\mathbf{R}}+\mathbf{Z}_{\mathbf{R D}} \\
\mathbf{Y}_{\mathbf{S D}} & =\mathbf{X}_{\mathbf{S}}+\mathbf{Z}_{\mathbf{S D}}
\end{aligned}
$$

where $\mathbf{X}_{\mathbf{S}}=\left[X_{1}, X_{2}, \cdots, X_{m}\right]^{\mathrm{T}}$ and $\tilde{\mathbf{X}}_{\mathbf{R}}=$ $\left[\tilde{X}_{m+1}, \tilde{X}_{m+2}, \cdots, \tilde{X}_{k}\right]^{\mathrm{T}}$ are the transmitted signal vectors from the source node and the relay node, respectively. $\mathbf{Y}_{\mathbf{S D}}=\left[Y_{1}, Y_{2}, \cdots, Y_{m}\right]^{\mathrm{T}}$ and $\tilde{\mathbf{Y}}_{\mathbf{S R}}=\left[\tilde{Y}_{1}, \tilde{Y}_{2}, \cdots, \tilde{Y}_{m}\right]^{\mathrm{T}}$ are the received signal vectors at the destination node and the relay node when the signal is transmitted from the source node. $\mathbf{Y}_{\mathbf{R D}}=\left[Y_{m+1}, Y_{m+2}, \cdots, Y_{k}\right]^{\mathrm{T}}$ is the received signal vector at the destination from the relay node. $\tilde{\mathbf{Z}}_{\mathbf{S R}}$ $=\left[\tilde{Z}_{1}, \tilde{Z}_{2}, \cdots, \tilde{Z}_{m}\right]^{\mathrm{T}}$ is the zero-mean independent additive white Gaussian noise (AWGN) vector with $E\left[\tilde{\mathbf{Z}}_{\mathbf{S R}} \tilde{\mathbf{Z}}_{\mathbf{S R}}^{\mathrm{T}}\right]$ $=\operatorname{diag}\left\{\tilde{N}_{1} / 2, \tilde{N}_{2} / 2, \cdots, \tilde{N}_{m} / 2\right\}$ at the relay node. $\mathbf{Z}_{\mathbf{S D}}$ $=\left[Z_{1}, Z_{2}, \cdots, Z_{m}\right]^{\mathrm{T}}$ is the zero-mean independent AWGN vector with $E\left[\mathbf{Z}_{\mathbf{S D}} \mathbf{Z}_{\mathbf{S D}}^{\mathrm{T}}\right]=\operatorname{diag}\left\{N_{1} / 2, N_{2} / 2, \cdots, N_{m} / 2\right\}$ at the destination node. $\mathbf{Z}_{\mathbf{R D}}=\left[Z_{m+1}, Z_{m+1}, \cdots, Z_{k}\right]^{\mathrm{T}}$ is the zero-mean independent AWGN vector with $E\left[\mathbf{Z}_{\mathbf{R D}} \mathbf{Z}_{\mathbf{R D}}^{\mathrm{T}}\right]=$ $\operatorname{diag}\left\{N_{m+1} / 2, N_{m+2} / 2, \cdots, N_{k} / 2\right\}$ at the destination. [.] $]^{\mathrm{T}}$ is the transpose operation of a vector.

Since the multiple channels are independent, the channel transition probability mass function is given by

$$
\begin{gathered}
P\left(y_{1}, \cdots, y_{k}, \tilde{y}_{1}, \cdots, \tilde{y}_{m} \mid x_{1}, \cdots, x_{m}, \tilde{x}_{m+1}, \cdots, \tilde{x}_{k}\right) \\
=\prod_{i=1}^{m} P\left(y_{i}, \tilde{y}_{i} \mid x_{i}\right) \prod_{j=m+1}^{k} P\left(y_{j} \mid \tilde{x}_{j}\right)
\end{gathered}
$$

For the channel transition probability mass function given in (2), we have the following capacity bounds for $(k, m)$-MBR.

Theorem 1: The upper and lower bounds for the capacity

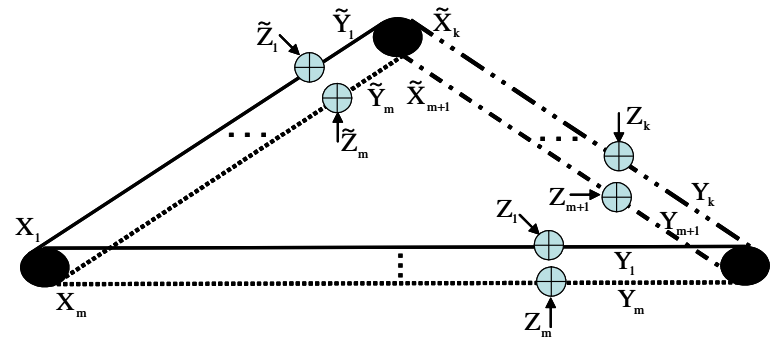

Fig. 1. $(k, m)$ Multi-Band Relay Channel

of $(k, m)$-MBR are given by

$$
\begin{aligned}
& C_{\text {low }}=\sup _{P(\cdot)} \min \left\{\sum_{i=1}^{m} I\left(X_{i} ; Y_{i}\right)+\sum_{i=m+1}^{k} I\left(\tilde{X}_{i} ; Y_{i}\right), \sum_{i=1}^{m} I\left(X_{i} ; \tilde{Y}_{i}\right)\right\} \\
& C_{\text {up }}=\sup _{P(\cdot)} \min \left\{\sum_{i=1}^{m} I\left(X_{i} ; Y_{i}\right)+\sum_{i=m+1}^{k} I\left(\tilde{X}_{i} ; Y_{i}\right), \sum_{i=1}^{m} I\left(X_{i} ; \tilde{Y}_{i}, Y_{i}\right)\right\}
\end{aligned}
$$

where $I(X ; Y)$ denotes the mutual information between $X$ and $Y$ and the input joint distribution $P(\cdot)$ is given by

$$
P\left(x_{1}, \cdots, x_{m}, \tilde{x}_{m+1}, \cdots, \tilde{x}_{k}\right)=P\left(x_{1}\right) \cdots P\left(\tilde{x}_{k}\right)
$$

\section{Achievable Rates and Optimum Resource ALLOCATION FOR $(3,2)$-MBR}

In this paper, we consider the hybrid wireless networks where the source node has access to distinct bands (standards) and a second node that overhears the source information relays to the destination using a separate band. We note that this case corresponds to the general case with $k-m=1$. It is readily seen that $k=2$ and $m=1$ corresponds to the Gaussian orthogonal relay channel considered in [8], [12]. When an additional system (orthogonal channel) becomes available for the source, we have the $(3,2)$-MBR. In the sequel, we will consider this tractable case and strategy that maximizes the capacity lower bound.

Under this network topology, we employ resource allocation to optimize the achievable rate for the case where the total available bandwidth is shared between the different systems involved. The input-output signal model is given by (1) with $k=3$ and $m=2$ under power constraints

$$
E\left[X_{i}^{2}\right] \leq \alpha_{i} P_{s}, \quad i=1,2
$$

where $P_{s}$ is the total available power at the source node, and $\alpha_{i}$ is the power allocation parameter for each orthogonal band. We assume that the relay node uses its available full power, $P_{r}$. We do not have a total power constraint between source and relay and assume each has its own battery.

The lower bound for the capacity of the $(3,2)$-MBR is

$$
\begin{array}{r}
C_{\text {low }}=\max _{0 \leq \alpha_{i}, \phi_{i} \leq 1} \min \left\{\sum_{i=1}^{2} \phi_{i} \log \left(1+\alpha_{i} \frac{\gamma_{s d}^{i}}{\phi_{i}}\right)+\phi_{3} \log \left(1+\frac{\gamma_{r d}^{3}}{\phi_{3}}\right)\right. \\
\left., \sum_{i=1}^{2} \phi_{i} \log \left(1+\alpha_{i} \frac{\gamma_{s r}^{i}}{\phi_{i}}\right)\right\}
\end{array}
$$

where all logarithms are base 2. Let $\sum_{i=1}^{2} \alpha_{i}=1$ and $\sum_{i=1}^{3} \phi_{i}$ 
$=1$ denote the power and bandwidth allocation parameters, respectively. We assume that the system has total bandwidth $W$ and the input and output signals are sampled every $1 / 2 W$ seconds. We define the SNR at the relay and destination over channel $i$ as,

$$
\gamma_{s r}^{i} \triangleq \frac{P_{s}}{\tilde{N}_{i} W}, \quad \gamma_{s d}^{i} \triangleq \frac{P_{s}}{N_{i} W}, \quad i=1,2
$$

and the received SNR at the destination over channel 3 as,

$$
\gamma_{r d}^{3} \triangleq \frac{P_{r}}{N_{3} W}
$$

Note that the actual received SNR values are scaled versions of (8) and (9) depending on the power and bandwidth allocation parameters. For example, the actual received SNR at the relay from channel 1 , which is allocated $\alpha_{1}$ fraction of the source power and $\phi_{1}$ fraction of the bandwidth, is simply $\alpha_{1} \gamma_{s r}^{1} / \phi_{1}$.

Given the received SNRs, which are assumed to be available at the source and relay, we can maximize the achievable rate by optimally choosing the power and the bandwidth. The optimum power and bandwidth choices are a function of the received SNRs at the relay and destination. We identify the optimum values for different ranges of SNRs. The following notation is used throughout the rest of the paper.

- We use $C_{\text {low1 }}$ and $C_{\text {low2 }}$ to indicate the multi-access cut and the broadcast cut of the lower bound.

- We denote $\alpha_{\text {Clow1 }}^{*}$ and $\alpha_{\text {Clow2 }}^{*}$ as the $\alpha$ that maximizes $C_{\text {low1 }}$ and $C_{\text {low2 }}$, respectively.

- We denote $\underline{\phi}_{\text {Clow1 }}^{*}$ and $\underline{\phi}_{\text {Clow2 } 2}^{*}$ as the $\left(\phi_{1}, \phi_{2}, \phi_{3}\right)$ that maximizes $C_{\text {low1 }}$ and $C_{\text {low2 } 2}$, respectively.

- Let $\alpha_{\text {int }}$ denote the $\alpha$ value where $C_{\text {low1 }}$ and $C_{\text {low2 }}$ intersect.

- Let $\phi_{\text {int }}$ denote the $\left(\phi_{1}, \phi_{2}, \phi_{3}\right)$ where $C_{\text {low1 }}$ and $C_{\text {low2 }}$ intersect.

- $A \cap B$ represents intersection of $\mathrm{A}$ and $\mathrm{B}$.

We now discuss how to maximize the achievable rate and find the associated optimum resource allocation parameters given the received SNRs. With the variable change $\alpha_{1}=\alpha$ and $\alpha_{2}$ $=1-\alpha$, the observations follow from the investigation of the graphical behavior of $C_{\text {low1 }}$ and $C_{\text {low2 } 2}$ as a function of $\alpha$.

Let us first note that when $\gamma_{s d}^{i} \geq \gamma_{s r}^{i}$ for $i=1,2$, the relay is not useful and we need to dedicate all resources to the direct link. When the conditions are not met, the max-min of (7) is more involved, and requires us to investigate the relationship between all dependent variables given in the following four Lemmas.

Lemma 1: For given $\gamma_{s d}, \gamma_{s r}, \gamma_{r d}$, and $\phi_{i}, i=1,2,3$ satisfying the following conditions:

$$
\begin{gathered}
\phi_{1} \log \left(1+\frac{\gamma_{s d}^{1}}{\phi_{1}}\right)+\phi_{3} \log \left(1+\frac{\gamma_{r d}^{3}}{\phi_{3}}\right)<\phi_{1} \log \left(1+\frac{\gamma_{s r}^{1}}{\phi_{1}}\right) \\
\phi_{2} \log \left(1+\frac{\gamma_{s d}^{2}}{\phi_{2}}\right)+\phi_{3} \log \left(1+\frac{\gamma_{r d}^{3}}{\phi_{3}}\right)>\phi_{2} \log \left(1+\frac{\gamma_{s r}^{2}}{\phi_{2}}\right)
\end{gathered}
$$

The maximum lower bound and its corresponding optimum resource allocation parameters are given by
Case 1: $\alpha_{\text {Clow1 }}^{*}<\alpha_{\text {int }}<\alpha_{\text {Clow2 }}^{*}$

$$
C_{\text {low }}=\max _{0 \leq \alpha, \phi_{i} \leq 1}\left(C_{\text {low1 }} \cap C_{\text {low } 2}\right),\left(\alpha^{*}, \underline{\phi}^{*}\right)=\left(\alpha_{\text {int }}, \underline{\text { int }}_{\text {int }}\right)
$$

Case 2: $\alpha_{\text {int }}<\alpha_{\text {Clow1 }}^{*}, \alpha_{\text {Clow2 }}^{*}$

$$
C_{\text {low }}=\max _{0 \leq \alpha, \phi_{i} \leq 1} C_{\text {low1 } 1}, \quad\left(\alpha^{*}, \underline{\phi}^{*}\right)=\left(\alpha_{\text {Clow1 }}^{*}, \underline{\phi}_{\text {Clow1 }}^{*}\right)
$$

Case 3: $\alpha_{\text {int }}>\alpha_{\text {Clow1 }}^{*}, \alpha_{\text {Clow2 }}^{*}$

$$
C_{\text {low }}=\max _{0 \leq \alpha, \phi_{i} \leq 1} C_{\text {low2 } 2}, \quad\left(\alpha^{*}, \underline{\phi}^{*}\right)=\left(\alpha_{\text {clow2 } 2}^{*}, \underline{\phi}_{\text {Clow2 }}^{*}\right)
$$

Proof: We note that (10) indicates that $C_{\text {low2 }}$ is larger than $C_{\text {low1 }}$ for $\alpha=1$. On the other hand, (11) means that $C_{\text {low2 }}$ is smaller than $C_{\text {low }}$ for $\alpha=0$. Since it is readily shown that for a fixed $\left(\phi_{1}, \phi_{2}, \phi_{3}\right), C_{\text {low } 1}$ and $C_{\text {low2 } 2}$ are strictly concave functions in $\alpha \in[0,1]$, we know that $C_{\text {low } 1}$ and $C_{\text {low } 2}$ must intersect at $\alpha_{\text {int }}$. There are three different possible cases for how they intersect. For case 1, the max-min of (7) can not be the maximum of $C_{\text {low1 }}$ or $C_{\text {low2 }}$, and must be the intersection at $\alpha^{*}=\alpha_{\text {int. }}$. For case 2, the max-min of (7) is $C_{\text {low1 }}$. Case 3 is the opposite of case 2 and the max-min of (7) is $C_{\text {low2 } 2}$. For each case, there is a set of feasible $\left(\phi_{1}, \phi_{2}, \phi_{3}\right)$ values satisfying (10) and (11). Therefore, for case 1 , the maximum lower bound is the maximum of possible intersection which leads to the corresponding optimum resource allocation parameters, $\left(\alpha_{\text {int }}, \phi_{\text {int }}\right)$. For case 2, the optimum resource allocation parameters are

$$
\left(\alpha_{\text {clowl }}^{*}, \underline{\phi}_{\text {Clow1 }}^{*}\right)= \begin{cases}\left(1,1-u_{1}, 0, u_{1}\right) & \text { if } \gamma_{s d}^{1}>\gamma_{s d}^{2} \\ \left(0,0,1-u_{2}, u_{2}\right) & \text { if } \gamma_{s d}^{1}<\gamma_{s d}^{2}\end{cases}
$$

where $u_{1}=\frac{\gamma_{r d}^{3}}{\gamma_{r d}^{3}+\gamma_{s d}^{1}}$ and $u_{2}=\frac{\gamma_{r d}^{3}}{\gamma_{r d}^{3}+\gamma_{s d}^{2}}$. For the case 2, the optimum resource allocation parameters are

$$
\left(\alpha_{\text {clow2 } 2}^{*}, \underline{\phi}_{\text {Clow2 }}^{*}\right)= \begin{cases}(1,1,0,0) & \text { if } \gamma_{s r}^{1}>\gamma_{s r}^{2} \\ (0,0,1,0) & \text { if } \gamma_{s r}^{1}<\gamma_{s r}^{2}\end{cases}
$$

Lemma 2: For given $\gamma_{s d}, \gamma_{s r}, \gamma_{r d}$, and $\phi_{i}, i=1,2,3$ satisfying the following conditions:

$$
\begin{gathered}
\phi_{1} \log \left(1+\frac{\gamma_{s d}^{1}}{\phi_{1}}\right)+\phi_{3} \log \left(1+\frac{\gamma_{r d}^{3}}{\phi_{3}}\right)>\phi_{1} \log \left(1+\frac{\gamma_{s r}^{1}}{\phi_{1}}\right) \\
\phi_{2} \log \left(1+\frac{\gamma_{s d}^{2}}{\phi_{2}}\right)+\phi_{3} \log \left(1+\frac{\gamma_{r d}^{3}}{\phi_{3}}\right)<\phi_{2} \log \left(1+\frac{\gamma_{s r}^{2}}{\phi_{2}}\right)
\end{gathered}
$$

The maximum lower bound and its corresponding optimum resource allocation parameters are given by

Case 1: $\alpha_{\text {Clow2 }}^{*}<\alpha_{\text {int }}<\alpha_{\text {clow1 }}^{*}$

$$
C_{\text {low }}=\max _{0 \leq \alpha, \phi_{i} \leq 1}\left(C_{\text {low1 }} \cap C_{\text {low } 2}\right),\left(\alpha^{*}, \underline{\phi}^{*}\right)=\left(\alpha_{\text {int }}, \underline{\phi}_{\text {int }}\right)
$$

Case 2: $\alpha_{\text {int }}<\alpha_{\text {Clow1 }}^{*}, \alpha_{\text {Clow2 }}^{*}$

$$
C_{\text {low }}=\max _{0 \leq \alpha, \phi_{i} \leq 1} C_{\text {low2 } 2}, \quad\left(\alpha^{*}, \underline{\phi}^{*}\right)=\left(\alpha_{\text {Clow2 } 2}^{*}, \underline{\phi}_{\text {Clow2 }}^{*}\right)
$$

Case 3: $\alpha_{\text {int }}>\alpha_{\text {Clow1 }}^{*}, \alpha_{\text {Clow2 }}^{*}$

$$
C_{\text {low }}=\max _{0 \leq \alpha, \phi_{i} \leq 1} C_{\text {lowl }}, \quad\left(\alpha^{*}, \underline{\phi}^{*}\right)=\left(\alpha_{\text {clowl }}^{*}, \underline{\phi}_{\text {Clow1 }}^{*}\right)
$$

Proof: We note that (17) indicates that $C_{\text {low } 2}$ is smaller than $C_{\text {low1 }}$ for $\alpha=1$. Also note that (18) implies that $C_{\text {low2 }}$ is 
larger than $C_{\text {lowl }}$ for $\alpha=0$. This indicates that Lemma 2 is the opposite of Lemma 1, and the proof is identical to that of Lemma 1 by switching the role of $C_{\text {low1 }}$ and $C_{\text {low2 }}$.

Lemma 3: For given $\gamma_{s d}, \gamma_{s r}, \gamma_{r d}$, and $\phi_{i}, i=1,2,3$ satisfying the following conditions:

$$
\begin{gathered}
\phi_{1} \log \left(1+\frac{\gamma_{s d}^{1}}{\phi_{1}}\right)+\phi_{3} \log \left(1+\frac{\gamma_{r d}^{3}}{\phi_{3}}\right)>\phi_{1} \log \left(1+\frac{\gamma_{s r}^{1}}{\phi_{1}}\right) \\
\phi_{2} \log \left(1+\frac{\gamma_{s d}^{2}}{\phi_{2}}\right)+\phi_{3} \log \left(1+\frac{\gamma_{r d}^{3}}{\phi_{3}}\right)>\phi_{2} \log \left(1+\frac{\gamma_{s r}^{2}}{\phi_{2}}\right)
\end{gathered}
$$

The maximum lower bound and its corresponding optimum resource allocation parameters are given by

Case 1: $\alpha_{\text {Clow2 }}^{*}<\alpha_{\text {int1 }}<\alpha_{\text {Clow1 }}^{*}$

$$
C_{\text {low }}=\max _{0 \leq \alpha, \phi_{i} \leq 1}\left(C_{\text {low1 }} \cap C_{\text {low2 }}\right),\left(\alpha^{*}, \underline{\phi}^{*}\right)=\left(\alpha_{\text {int1 }}, \underline{\phi}_{\text {int }}\right)
$$

Case 2: $\alpha_{\text {Clow1 }}^{*}<\alpha_{\text {int2 }}<\alpha_{\text {Clow2 }}^{*}$

$$
C_{\text {low }}=\max _{0 \leq \alpha, \phi_{i} \leq 1}\left(C_{\text {low1 }} \cap C_{\text {low } 2}\right),\left(\alpha^{*}, \underline{\phi}^{*}\right)=\left(\alpha_{\text {int2 }}, \underline{\phi}_{\text {int }}\right)
$$

Case 3: $\alpha_{\text {int1 }}>\alpha_{\text {Clow1 }}^{*}, \alpha_{\text {Clow2 }}^{*}$ and $\alpha_{\text {int } 2}<\alpha_{\text {Clow1 }}^{*}, \alpha_{\text {Clow2 }}^{*}$

$$
C_{\text {low }}=\max _{0 \leq \alpha, \phi_{i} \leq 1} C_{\text {lowl }}, \quad\left(\alpha^{*}, \underline{\phi}^{*}\right)=\left(\alpha_{\text {Clow1 }}^{*}, \underline{\phi}_{\text {Clow1 }}^{*}\right)
$$

Case 4: $\alpha_{\text {int }}=$ NULL

$$
C_{\text {low }}=\max _{0 \leq \alpha, \phi_{i} \leq 1} C_{\text {low } 2}, \quad\left(\alpha^{*}, \underline{\phi}^{*}\right)=\left(\alpha_{\text {Clow2 } 2}^{*}, \underline{\phi}_{\text {Clow } 2}^{*}\right)
$$

Proof: We note that (22) and (23) indicate that $C_{\text {low2 }}$ is smaller than $C_{\text {low } 1}$ for $\alpha=0$ and 1 . Thus, there must exist two intersections at $\alpha_{\text {int1 }}$ and $\alpha_{\text {int2 }}\left(\alpha_{\text {int1 }}>\alpha_{\text {int2 }}\right)$. Otherwise, there is no intersection at all. When there is no intersection, that corresponds to case 4 , and, it is readily shown that $C_{\text {low } 1}$ is always larger than $C_{\text {low2 }}$ over $\alpha \in[0,1]$. Therefore, the max$\min$ of (7) is $C_{\text {low2 }}$. When they intersect, there are three different possible cases. For case 1, we observe that the intersection value at $\alpha_{\text {int }}$ is smaller than the maximum of $C_{\text {low1 }}$ or $C_{\text {low2 } 2}$. Thus, the max-min of (7) must be the intersection value at $\alpha^{*}=\alpha_{\text {int } 1}$. For case 2, we observe that the intersection value at $\alpha_{\text {int2 }}$ is smaller than the maximum of $C_{\text {low1 }}$ and $C_{\text {low2 }}$. Thus, max-min of (7) must be the intersection value at $\alpha^{*}=\alpha_{\text {in2 } 2}$. For case 3, the maximum value of $C_{\text {low1 }}$ and $C_{\text {low } 2}$ are larger than two intersection values. Therefore, the max-min of (7) is $C_{\text {low1 }}$.

Lemma 4: For given $\gamma_{s d}, \gamma_{s r}, \gamma_{r d}$, and $\phi_{i}, i=1,2,3$ satisfying the following conditions:

$$
\begin{aligned}
\phi_{1} \log \left(1+\frac{\gamma_{s d}^{1}}{\phi_{1}}\right)+\phi_{3} \log \left(1+\frac{\gamma_{r d}^{3}}{\phi_{3}}\right)<\phi_{1} \log \left(1+\frac{\gamma_{s r}^{1}}{\phi_{1}}\right) \\
\phi_{2} \log \left(1+\frac{\gamma_{s d}^{2}}{\phi_{2}}\right)+\phi_{3} \log \left(1+\frac{\gamma_{r d}^{3}}{\phi_{3}}\right)<\phi_{2} \log \left(1+\frac{\gamma_{s r}^{2}}{\phi_{2}}\right)
\end{aligned}
$$

The maximum lower bound and its corresponding optimum resource allocation parameters are given by

Case 1: $\alpha_{\text {Clow1 }}^{*}<\alpha_{\text {int1 }}<\alpha_{\text {Clow2 }}^{*}$

$$
C_{\text {low }}=\max _{0 \leq \alpha, \phi_{i} \leq 1}\left(C_{\text {low1 }} \cap C_{\text {low } 2}\right),\left(\alpha^{*}, \underline{\phi}^{*}\right)=\left(\alpha_{\text {int1 }}, \underline{\phi}_{\text {int }}\right)
$$

TABLE I

Four SCENARIOS DEPENDING ON CHANNEL CONDITIONS

\begin{tabular}{|l|c|c|c|c|}
\hline Scenario & I & II & III & IV \\
\hline \hline SR & C $>$ N & C $<\mathrm{N}$ & $\mathrm{C}<\mathrm{N}$ & $\mathrm{C}>\mathrm{N}$ \\
\hline SD & $\mathrm{C}>\mathrm{N}$ & $\mathrm{C}<\mathrm{N}$ & $\mathrm{C}>\mathrm{N}$ & $\mathrm{C}<\mathrm{N}$ \\
\hline
\end{tabular}

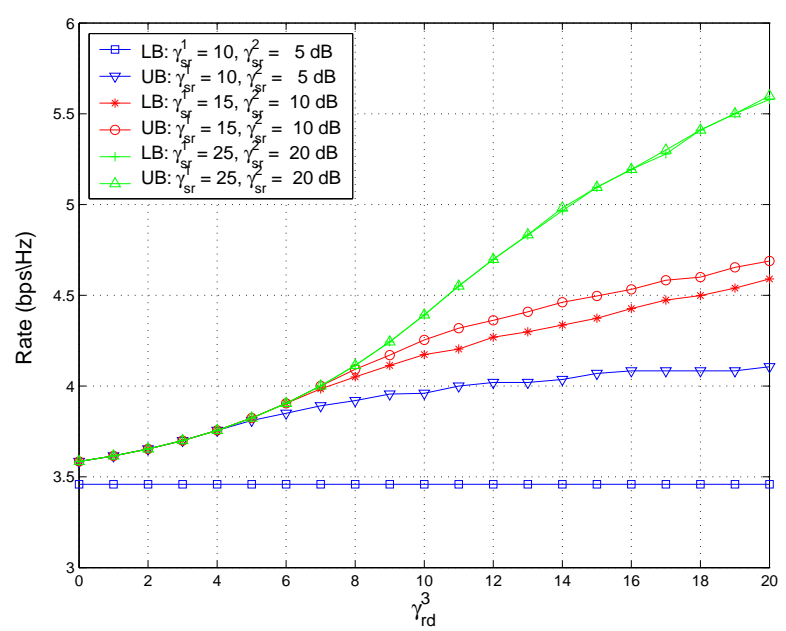

Fig. 2. The upper and lower bounds for $(3,2)-\mathrm{MBR}$ with $\gamma_{s d}^{1}=10 \mathrm{~dB}$ and $\gamma_{s d}^{2}=5 \mathrm{~dB}$

Case 2: $\alpha_{\text {Clow2 }}^{*}<\alpha_{\text {int2 }}<\alpha_{\text {Clow1 }}^{*}$

$$
C_{\text {low }}=\max _{0 \leq \alpha, \phi_{i} \leq 1}\left(C_{\text {low } 1} \cap C_{\text {low } 2}\right),\left(\alpha^{*}, \underline{\phi}^{*}\right)=\left(\alpha_{\text {int }}, \underline{\phi}_{\text {int }}\right)
$$

Case 3: $\alpha_{\text {int1 }}>\alpha_{\text {Clow1 }}^{*}, \alpha_{\text {Clow2 }}^{*}$ and $\alpha_{\text {int2 }}<\alpha_{\text {Clow1 }}^{*}, \alpha_{\text {Clow2 }}^{*}$

$$
C_{\text {low }}=\max _{0 \leq \alpha, \phi_{i} \leq 1} C_{\text {low2 } 2}, \quad\left(\alpha^{*}, \underline{\phi}^{*}\right)=\left(\alpha_{\text {clow } 2}^{*}, \underline{\phi}_{\text {Clow } 2}^{*}\right)
$$

Case 4: $\alpha_{\text {int }}=$ NULL

$$
C_{\text {low }}=\max _{0 \leq \alpha, \phi_{i} \leq 1} C_{\text {low1 }}, \quad\left(\alpha^{*}, \underline{\phi}^{*}\right)=\left(\alpha_{\text {clowl }}^{*}, \underline{\phi}_{\text {Clow1 }}^{*}\right)
$$

Proof: We note that the inequalities (28) and (29) indicate that $C_{\text {low2 }}$ is larger than $C_{\text {low } 1}$ for $\alpha=0$ and 1 . This indicates that Lemma 4 is the opposite of Lemma 3, and the proof is identical to that of Lemma 3 by switching the role of $C_{\text {low } 1}$ and $C_{\text {low2} 2}$.

\section{UPPER BOUND ON CAPACITY}

So far, we considered the maximization of the achievable rate, i.e, the lower bound on the capacity of the MBR. In this section, we consider the capacity upper bound and its maximization. The upper bound of $(3,2)$-MBR is given by

$$
\begin{array}{r}
C_{\text {up }}=\max _{0 \leq \alpha, \phi_{i} \leq 1} \min \left\{\sum_{i=1}^{2} \phi_{i} \log \left(1+\alpha_{i} \frac{\gamma_{s d}^{i}}{\phi_{i}}\right)+\phi_{3} \log \left(1+\frac{\gamma_{r d}^{3}}{\phi_{3}}\right)\right. \\
\left., \sum_{i=1}^{2} \phi_{i} \log \left(1+\alpha_{i} \frac{\gamma_{s d}^{i}+\gamma_{s r}^{i}}{\phi_{i}}\right)\right\}
\end{array}
$$

We note that the upper bound is obtained by the max-flow min-cut theorem and the lower bound given in (7) is achieved by block Markov coding. In general, these bounds are not tight. The block Markov coding requires the relay to decode 


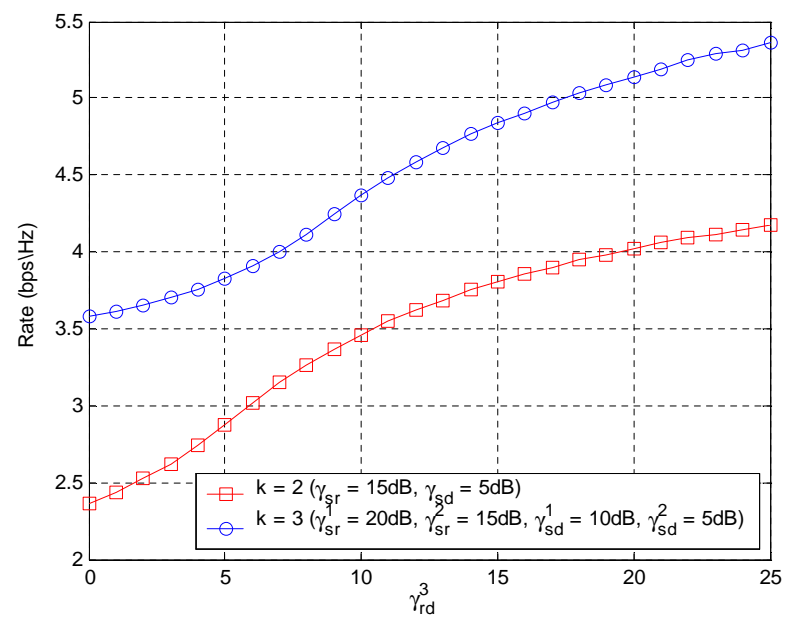

Fig. 3. Comparison of achievable rates for scenario II

the whole block [12]. Thus, if the source to relay link is poor, the decoding error will be high enough to propagate error. On the other hand, if the source to relay link is much better than source to destination link, the bounds become tighter and the capacity can be found. We can observe this by comparing (34) and (7). As $\gamma_{s r}^{1}$ and $\gamma_{s r}^{2}$ gets much larger than $\gamma_{s d}^{1}$ and $\gamma_{s d}^{2}$, the two maximized bounds coincide and yield the maximum capacity. This is illustrated in the Figure 2.

The maximization of the upper bound follows similar steps to that of the lower bound. For a fixed $\left(\phi_{1}, \phi_{2}, \phi_{3}\right)$, the broadcast cut of the upper bound is a concave function in $\alpha \in[0,1]$. Therefore, we have the equivalent four Lemmas to those in Section III, depending on the four different inequality conditions of the multi-access cut and the broadcast cut at $\alpha=0$ and 1 as follows.

$\phi_{1} \log \left(1+\frac{\gamma_{s d}^{1}}{\phi_{1}}\right)+\phi_{3} \log \left(1+\frac{\gamma_{r d}^{3}}{\phi_{3}}\right) \lessgtr \phi_{1} \log \left(1+\frac{\gamma_{s d}^{1}+\gamma_{s r}^{1}}{\phi_{1}}\right)$

$\phi_{2} \log \left(1+\frac{\gamma_{s d}^{2}}{\phi_{2}}\right)+\phi_{3} \log \left(1+\frac{\gamma_{r d}^{3}}{\phi_{3}}\right) \lessgtr \phi_{2} \log \left(1+\frac{\gamma_{s d}^{2}+\gamma_{s r}^{2}}{\phi_{2}}\right)$

Let us denote $\alpha_{\text {Cup1 }}^{*}$ and $\alpha_{\text {Cup } 2}^{*}$ as the $\alpha$ that maximizes $C_{\text {up } 1}$ and $C_{\text {up } 2}$, respectively. We also denote $\underline{\phi}_{\text {Cup1 }}^{*}$ and $\underline{\phi}_{\text {Cup } 2}^{*}$ as the $\left(\phi_{1}, \phi_{2}, \phi_{3}\right)$ that maximizes $C_{\text {up1 }}$ and $C_{\text {up2 }}$, respectively. We note that multi-access cut of the lower and upper bound are the same. Thus, the optimum resource allocation parameters $\left(\alpha_{\text {Cupl }}^{*}, \phi_{\text {Cupl }}^{*}\right)$ are the same as $\left(\alpha_{\text {Clow1 }}^{*}, \phi_{\text {Clow1 }}^{*}\right)$. On the other hand, the optimum resource allocation parameters that maximize the broadcast cut are given by

$$
\left(\alpha_{\text {Cup } 2}^{*}, \underline{\phi}_{\text {Cup } 2}^{*}\right)= \begin{cases}(1,1,0,0) & \text { if } \gamma_{s d}^{1}+\gamma_{s r}^{1}>\gamma_{s d}^{2}+\gamma_{s r}^{2} \\ (0,0,1,0) & \text { if } \gamma_{s d}^{1}+\gamma_{s r}^{1}<\gamma_{s d}^{2}+\gamma_{s r}^{2}\end{cases}
$$

\section{RESUlts AND Discussion}

When a new wireless link becomes available at the source in addition to the existing single band relay network, a hybrid wireless network can be formed. In this case, a significant question to be answered is how to allocate resources between links in order to maximize the data rate. It is evident that the resource allocation strategy is a function of the channel

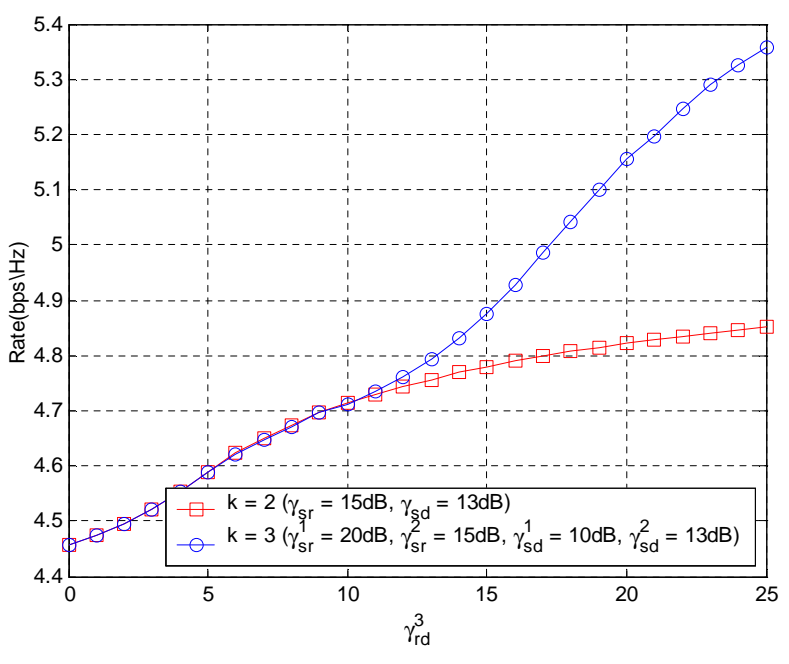

Fig. 4. Comparison of achievable rates for scenario III

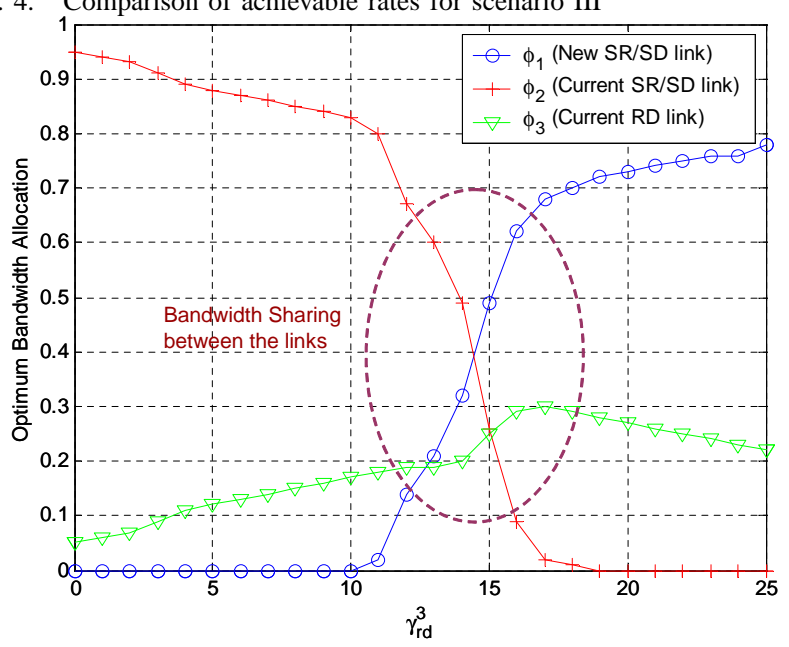

Fig. 5. Optimum bandwidth allocation for $K=3$ of scenario III

quality of each of the available links (SD/SR/RD). To answer this question, we consider four different scenarios depending on the relative quality of channel conditions summarized in Table I. In Table I, C and $\mathrm{N}$ stand for the current link and the new link, respectively. For each scenario, we compare the achievable rate and resource allocation strategy for $k=2,3$. That is, we aim to observe the effect of adding a new link, e.g. from $k=2$ to $k=3$ on the maximum achievable rate under four different scenarios.

Figure 3 shows the achievable rate for scenario II where channel condition of the SR link and the SD link of the newly added link is better than that of the SD link and the SR link of the current link. Comparing $k=3$ and $k=2$, we observe that the achievable rate of $k=3$ is better than that of $k=2$. This is because quality of the new link is better than that of the current link, and all resources are allocated to the better link. On the other hand, for scenario I where the channel condition of the SR link and the SD link of the newly added link is worse than that of the SD link and the SR link of the current link, the maximum achievable rates stays the same because all resources are allocated to the current link.

Figure 4 shows the achievable rate for scenario III where the new SR link is better than the current SR link, and the 


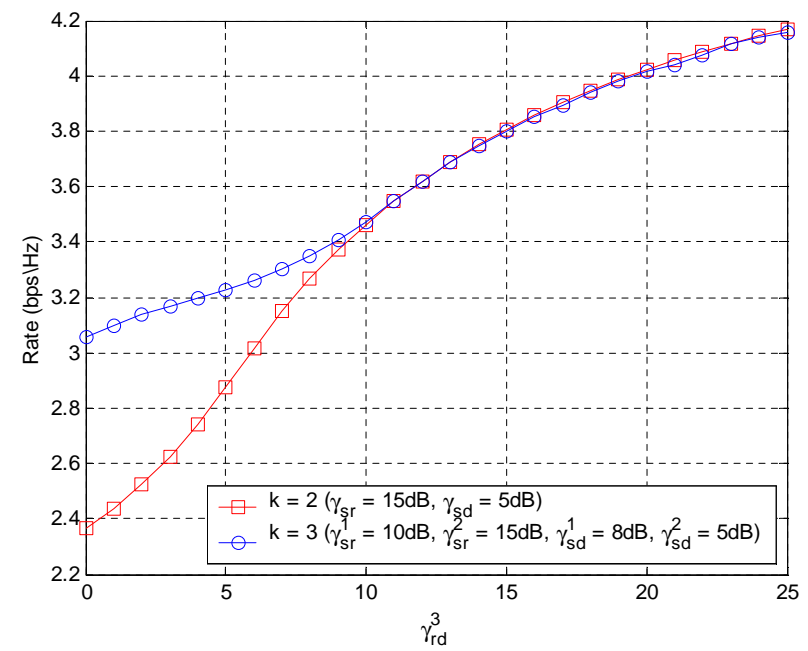

Fig. 6. Comparison of achievable rates for scenario IV

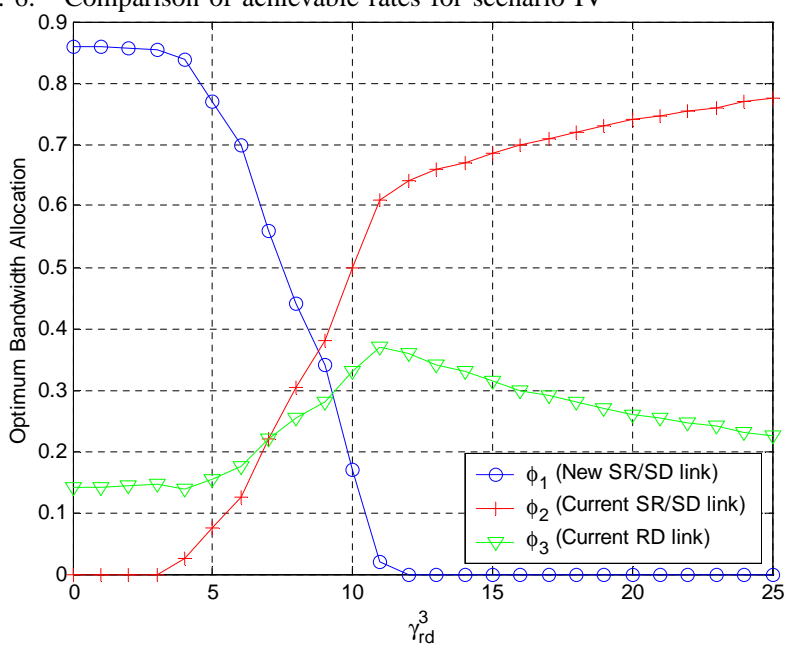

Fig. 7. Optimum bandwidth allocation for $K=3$ of scenario IV

new SD link is worse than the current SD link. We observe that the achievable rate for $k=2,3$ is almost same for low RD SNR. This is because when the RD link is not good, the relay becomes less useful, and most of bandwidth and power are allocated into channel with the best direct link. As the RD SNR increases, we observe that the achievable rate for $k=3$ is larger than that of $k=2$. This is because it is optimum resource allocation that we allocate more bandwidth and power to the new link with the best SR link.

The above observation is justified by examining bandwidth allocation (the power allocation follows a similar pattern) for $k=3$ shown in Figure 5. We observe that more bandwidth is allocated to the current link ( $\phi_{2}$ for $k=3$ ) for low received RD SNR. More bandwidth is allocated to the new link ( $\phi_{1}$ for $k=3$ ) when the RD link becomes better.

Figure 6 shows the achievable rate for scenario IV where the new SR link is worse than the current SR link and the new SD link is better than the current SD link. We note that this case is the opposite case to the scenario III. Thus, the achievable rate of $k=3$ is larger than that of $k=2$ when we have relatively poor RD link. Similarly, the achievable rate of the two cases become the same when we have a good RD link because more resources are allocated into the better current SR link.

Figure 7 shows the corresponding bandwidth allocation for $k=3$. We observe that more bandwidth is allocated to the new link when the received RD SNR is low because the new direct link is better. It is, however, seen that more bandwidth is allocated to the current link when the RD link becomes better because the current SR link is better than that of new link.

\section{CONCLusions}

In this paper, we have investigated the information-theoretic performance of a simple hybrid wireless network where the source, with the help of a relay node, communicates to the destination via multiple orthogonal channels. We have considered the case where the total bandwidth is dynamically allocated between the multiple channels from the source and the relay in addition to the source power. In particular, we have derived the optimum power and bandwidth allocation parameters in order to maximize the achievable rate.

Our numerical results have investigated the scenario where a new link at the source becomes available for an existing frequency division relay network, and the power and bandwidth resources are to be reallocated. We observe that the source node is encouraged to communicate over the best link by dedicating all resource when the new SR link and SD link are better (or worse) than that of the current SD link and SR link. Otherwise, it is beneficial to share resource between the current link and the new link to achieve the higher rate.

The simple hybrid wireless network investigated in this paper can be considered as a building block for more complex hybrid wireless networks. From the system design point of view, we conclude that, for this simple network, higher achievable rates can be obtained by optimally allocating resources between multiple standards.

\section{REFERENCES}

[1] H. Luo, R. Ramjee, P. Sinha, L. Li, and S. Lu. UCAN : A unified cellular and ad-hoc network architecture. In ACM MOBICOM'03, pages $353-$ 367, September 2003.

[2] E. C. van der Meulen. Three terminal communications channels Advanced Applied Probability, 3(5):120 - 154, September 1971.

[3] T. M. Cover and A. A. El Gamal. Capacity theorems for the relay channel. IEEE Transactions on Information Theory, 25(5):572 - 584, September 1979.

[4] G. Kramer, M. Gastpar, and P. Gupta. Cooperative strategies and capacity theorems for relay networks. Submitted to IEEE Transactions on Information Theory (revised), November 2004.

[5] B. Schein and R. G. Gallager. The Gaussian parallel relay network. In IEEE International Symposium on Information Theory, ISIT 00', page 22, 2000.

[6] P. Gupta and P. R. Kumar. Towards an information theory of large networks: an achievable rate region. IEEE Transactions on Information Theory, 49(8):1877 - 1894, August 2003.

[7] J. N. Laneman, D. N. C. Tse, and G. W. Wornell. Cooperative diversity in wireless networks: Efficient protocols and outage behavior. IEEE Transactions on Information Theory, 50(12):3062 - 3080, December 2004.

[8] Y. Liang and V. V. Veeravalli. Gaussian orthogonal relay channel: optimal resource allocation and capacity. Submitted to IEEE Transactions on Information Theory (revised), January 2005.

[9] A. Høst-Madsen and J. Zhang. Capacity bounds and power allocation in wireless relay channel. To appear in IEEE Transactions on Information Theory, June 2005

[10] I. Maric and R. D. Yates. Forwarding strategies for Gaussian parallelrelay networks. In Conference on Information Sciences and Systems, CISS' 04, pages 591 - 596, March 2004. 
[11] R. U. Nabar, H. Bölcskei, and F. W. Kneubühler. Fading relay channels: performance limits and space-time signal design. IEEE Journal on Selected Areas in Communications, 22(6):1099 - 1109, August 2004.

[12] A. A. El Gamal, M. Mohseni, and S. Zahedi. On reliable communication over Additive White Gaussian Noise relay channels. Submitted to IEEE Transactions on Information Theory, September 2004. 